\title{
Protocol
}

\section{A quasi-experimental study on exploring the use of mobile phone technology for optimizing, tracking and responding to children's developmental progress in Korogocho, Nairobi, Kenya: study protocol}

\author{
Patricia Kitsao Wekulo, Margaret Nampijja*, Domnick O. Okullo, Kenneth O. Okelo, \\ Milka Njeri, Silas Onyango, Elizabeth Kimani Murage
}

Maternal and Child Wellbeing Unit, African Population and Health Research Center, APHRC Campus, Manga Close, Off Kirawa Road, Nairobi, Kenya

Received: 18 September 2019

Accepted: 22 November 2019

\section{*Correspondence:}

Dr. Margaret Nampijja,

E-mail: maggie.nampijja@gmail.com

Copyright: (c) the author(s), publisher and licensee Medip Academy. This is an open-access article distributed under the terms of the Creative Commons Attribution Non-Commercial License, which permits unrestricted non-commercial use, distribution, and reproduction in any medium, provided the original work is properly cited.

\begin{abstract}
Background: The massive use of technology can be leveraged to facilitate access to growth and development programs for children. Existing programs supporting such initiatives for children younger than three years are inadequate and not accessible to most families. In most cases, primary caregivers are unable to identify delayed milestones in their children's growth and development due to inadequate information. They therefore often report the cases when they have become very severe and difficult to reverse. In order to promote early identification of possible developmental delays, African Population and Health Research Center together with Val Partners will develop, implement and evaluate the use of mobile phone technology to help caregivers track their children's developmental outcomes.

Methods: The study will employ a quasi-experimental design and will use a mixed-methods approach combining quantitative and qualitative methodologies. In one arm, 110 caregivers will be trained on the use of a mobile phone application to assess child growth and development. The other arm, with 110 caregivers, will receive standard care provided by community health volunteers. Child developmental outcomes will be assessed in both arms. Feasibility of the intervention will be assessed qualitatively. Performance data will be compared across the two arms using mixed linear models to assess the effect of the intervention on child development.

Conclusions: The findings are expected to provide evidence on whether the intervention is feasible and has an effect on child developmental outcomes. The results will inform the scalability and sustainability of the project.

Trial Registration: The trial has been registered with the Pan African Clinical Trial Registry (www.pactr.org) database (ID number: PACTR201905787868050).
\end{abstract}

Keywords: Child developmental outcomes, Mobile phone technology, Monitoring, Feasibility

\section{INTRODUCTION}

It is estimated that up to $95 \%$ of the global population lives in areas that have mobile-cellular network coverage. ${ }^{1}$ Mobile phone technology has drastically improved communication among people worldwide and especially in developing countries. ${ }^{1}$ Like in several other developing countries, mobile phone use in Kenya has increased considerably and many families now own mobile phones. ${ }^{2,3}$ The massive use of technology can be leveraged to facilitate children's growth and development especially in settings where programs supporting children's growth and development, particularly for those younger than three years, may be inadequate and not accessible to many families. ${ }^{4}$ 
Notably, about 250 million (43\%) children under five years in low- and middle-income countries (LMICs) are at risk of not achieving their developmental potential. ${ }^{5}$ The proportion of at-risk children is higher in subSaharan Africa (SSA), at $67 \%$, due to exposure to multiple risks including poverty, malnutrition, poor health, and unstimulating environments. ${ }^{6}$ The situation is more critical in informal settlements where residents are disadvantaged in many ways: they are faced with high levels of poverty and food insecurity; and, their children are more frequently sick and experience higher child malnutrition and mortality rates than other subpopulations. ${ }^{7}$ In many urban informal settlements, one of the most salient poverty-related caregiving challenges that caregivers face is the provision of appropriate stimulation of children. ${ }^{8}$ Residents in urban informal settlements tend to become parents at a much earlier age, with over half of mothers of children under five years being aged below 25 years; about $25 \%$ are adolescents. Education levels among such caregivers are lower than among those in the upper socio-economic divides of the population. Low parental education and young parenting age have been associated with poor caregiving, malnutrition and poor health in children. ${ }^{5}$

It is essential to empower primary caregivers to be able to care for and stimulate their children for optimal growth and development. Such an intervention should include equipping primary caregivers with basic knowledge on how to assess any delays in their children's development in the course of daily activities for immediate action. ${ }^{9}$ For many interventions among children, priority is often assigned to disorders that have a high prevalence, and those which are associated with developmental trajectories that could result in adverse behavioral, social, and psychological outcomes. ${ }^{9}$ Even though early assessment is important, there is a shortage of appropriate assessment/screening tools to identify developmental delays contextualized to SSA, hampering efforts to identify and adequately monitor at-risk children. ${ }^{10}$ In most cases, primary caregivers are unable to identify delayed milestones in their children's growth and development due to inadequate information on how to assess the key developmental milestones. By the time they report such cases, most of them would have become very severe. To mitigate such situations, parents should be sensitized on how to provide adequate care for development but also be able to detect early signs of developmental delay or impairment. ${ }^{11}$ As children's development takes place through daily life activities, caregivers should be able to pick up on early signs of developmental delay during their regular interaction and seek advice in real time.

We propose a project which will utilize mobile phone technology to help primary caregivers to identify, track and respond to delays and challenges in their children's development in a timely manner. Primary caregivers (particularly young mothers) in Nairobi will be trained on how to record developmental milestones achieved by their children in real time, in order to help them identify and address developmental delays early. The innovation will rely on the use of a short messaging system (SMS) where caregivers will receive messages on the expected achievement of a developmental milestone and send feedback in real time. Community health volunteers (CHVs) will carry out home visits to check on the progress of the child, and whether the caregiver is responding to the messages appropriately.

\begin{abstract}
Aim
The proposed study aims to develop and test the feasibility of using a mobile application by primary caregivers to track their children's developmental progress in informal settlements in Nairobi, as well as measure its effect on child development. The proposed work will provide a simple and user-friendly platform for measurement and tracking of key child development indicators. The data generated will provide useful information for development of an up-to scale program for children in the entire country and in similar low- and middle-income populations.
\end{abstract}

\section{METHODS}

\section{Study design}

The study will employ a quasi-experimental design and will use a mixed methods approach combining quantitative surveys, qualitative studies, and direct measurements. The study will comprise two arms: the intervention arm in which participants (caregivers and their children) will be trained on the use of the mobile technology and receive monthly text messages and advice about their children's development; and the control arm in which caregivers will receive the standard of care that is provided within the community by CHVs. The allocation of study participants will be based on the locations (villages) within the study site. The selected villages will be randomized to either the intervention or control arm and caregivers residing within the respective villages will belong to the respective arm. At baseline (before the intervention), we will collect information on socio-demographic characteristics, caregiver knowledge and practices on childcare and development, child growth and the developmental stages of the children. The intervention will then be administered for a year. At the end, we will measure and compare the outcomes across the two arms using quantitative and qualitative tools.

\section{Study setting}

The study will be conducted in Korogocho ward within Ruaraka sub-County, in Nairobi County, Kenya. Nairobi County which has 17 sub-counties is one of the 47 counties in Kenya. Each sub-County is further divided into wards for ease of administration. According to the 2014 national statistics, Nairobi County has a total population size of about $4,004,400 .^{12}$ 
Korogocho is a densely populated ward with 41,946 residents, and is characterized by high levels of poverty, high fertility rate and a significant proportion of young mothers; 25 per cent of mothers of children under five are aged less than 20 years. ${ }^{13}$ Poor feeding and child care practices have been documented among these mothers, ${ }^{8}$ and are associated with a high stunting rate $(50 \%)$ and high infant mortality rate of 57 deaths per 1000 live births. $^{7}$ Young mothers lack appropriate childcare knowledge and have limited ability to make childcare decisions; they typically rely on their mothers and other older women for guidance. ${ }^{8}$ Moreover, they have limited access to professional advice about childcare due to self and social discrimination. These factors, together with extreme poverty levels, hamper these young mothers' ability to provide the necessary stimulation for their children.

Korogocho ward, where over 2000 mothers of children under five are aged 15 to 19 years (unpublished data), provides an ideal setting for testing an intervention for enabling women to actively monitor and respond to their children's development in real time. Two groups of villages located at the extreme ends of Korogocho will be included in the study. Caregivers and their children residing in these villages and meeting the criteria will be invited to participate. The two groups will be randomly assigned to either intervention or control arms of the study, and the villages between them will act as a buffer zone to avoid contamination across the two allocations.

\section{Study population}

The project will target young mothers (including adolescents) of children under the age of two years and living in Korogocho. Efforts will be made to ensure equal representation of genders in the intervention as both fathers and mothers will be included during all communications and activities related to the project.

Primary caregivers and their young children aged between 6 and 24 months residing in selected villages of Korogocho will be recruited into the study. In each village, households with children in the eligible agegroup will be identified using the Nairobi Urban Health and Demographic Surveillance System (NUHDSS) database. The NUHDSS is conducted annually by the African Population and Health Research Center (APHRC) in Korogocho ward. A list of all potential participants will be generated and the required number will be randomly selected and approached about the study. We will work with CHVs within the respective villages to locate the mothers/ primary caregivers and to invite them to participate.

\section{Inclusion and exclusion criteria}

Primary caregivers will be eligible to participate if they have a child aged between 6 and 24 months, are willing to participate in the study and will be resident in
Korogocho for at least one year. Only primary caregivers with access to a phone will be considered for the intervention arm.

Any short-term residents (less than one year) will be excluded from the study. Children above two years will also be excluded from the study. Also excluded will be women (or men) incapable of giving consent (e.g. those with mental illness).

\section{Sample size calculation}

The sample size was calculated using $G *$ Power program where the program was set to a one-sided t-test involving the difference between two independent means. ${ }^{14}$ Using a priori power analysis, we input the values of 0.05 and 0.84 for significance levels and power, respectively. Additionally, equal-sized sample groups are assumed, meaning the allocation ratio of $\mathrm{N} 1$ (intervention group) to $\mathrm{N} 2$ (control group) is 1 . The calculation produced a sample size of 100 for each group, which allowing for a $10 \%$ attrition rate gave a result of 110 . We therefore hypothesize that with 100 caregiver-infant dyads in the intervention group and 100 in the control group, the sample size achieves $84 \%$ power to detect a difference among the two groups, with an effect size of $r=0.3$, at the $5 \%$ significance level (one-tailed test). A sample comprising a total of 220 households (110 caregiver-child dyads in each arm) will therefore be recruited.

\section{The intervention}

APHRC in partnership with Val Partners, will develop an ECD mobile phone technology application to help young mothers track and respond to their children's developmental progress in a timely manner. The proposed intervention focusses on improving caregivers' knowledge, attitudes and practices on children's growth and development through training, use of mobile phone technology to track and stimulate their children's growth and development, identifying developmental delays, mentorship visits by $\mathrm{CHV}_{\mathrm{s}}$ and referral to a health facility to address the identified developmental delays.

The specific intervention will be developed through the following steps (Figure 1).

Co-designing the mobile application with the caregivers and CHVs: In the first few months of the project, the project team will co-design the mobile application with caregivers and other stakeholders involved in the project that will enable primary caregivers to track and respond to developmental progression of their children. Appropriate child stimulation messages will initially be created together with caregivers, through focus group discussions to establish the type of messages to be delivered to caregivers based on their current caregiving practices, and social and cultural contexts. This information will be used to develop, with other partners, 
a health-messaging platform for use with mothers or caregivers.

Training of the CHVs and the caregivers: CHVs and caregivers will be trained on how to use the mobile application to record achievement of milestones and track children's growth and development.

Caregivers will receive monthly stimulation messages on play and communication activities to engage in to enhance their children's development. The mobile phone technology innovation will be programed to generate customized child stimulation messages based on the age of the child and previous feedback.

Home visits: These will be conducted monthly over a period of approximately 12 months. CHVs will visit the homes regularly to establish if the child stimulation messages received by caregivers are being put into practice.

Referrals and connections to other services for families in the areas of health and nutrition: When the child's development is delayed, this will be flagged on the mobile app system and a message will be sent to the assigned $\mathrm{CHV}$ for action. The $\mathrm{CHV}$ will then visit that particular household so that s/he can provide the necessary support to the caregiver to address the delay.

The overall solution structure utilizes existing CHVs to link with caregivers in marginalized communities to track and capture early childhood development indicators, and respond to developmental delays in a timely manner. The intervention is based on training and mentoring $\mathrm{CHV}_{\mathrm{s}}$ on the use of mobile technology to track children's growth and development and to identify key development delays. The CHVs will visit households to train caregivers on how to assess their children's growth and development, how to stimulate their growth and how to respond to the messages received on mobile phone technology to report any developmental delays. Since $\mathrm{CHV}_{\mathrm{s}}$ are part of the existing health system in Kenya, their regular interactions with mothers/ primary caregivers enhance community acceptance.

\section{The control arm}

Caregivers and their children in the control arm will receive the standard care provided by the ministry of health through CHVs. In this system, CHVs are recruited and trained in supporting and coordinating health activities in the communities where they live. Each CHV is allocated specific households which they are responsible for. CHVs follow up on health, growth, nutrition, and development of children under five years and advise or refer as necessary. They also monitor immunization status, and administer routine deworming tablets and vitamin A supplements. They participate in community mobilization and sensitizations and reporting in case of disease outbreaks. CHVs support various non- government health programs that are conducted within the community.

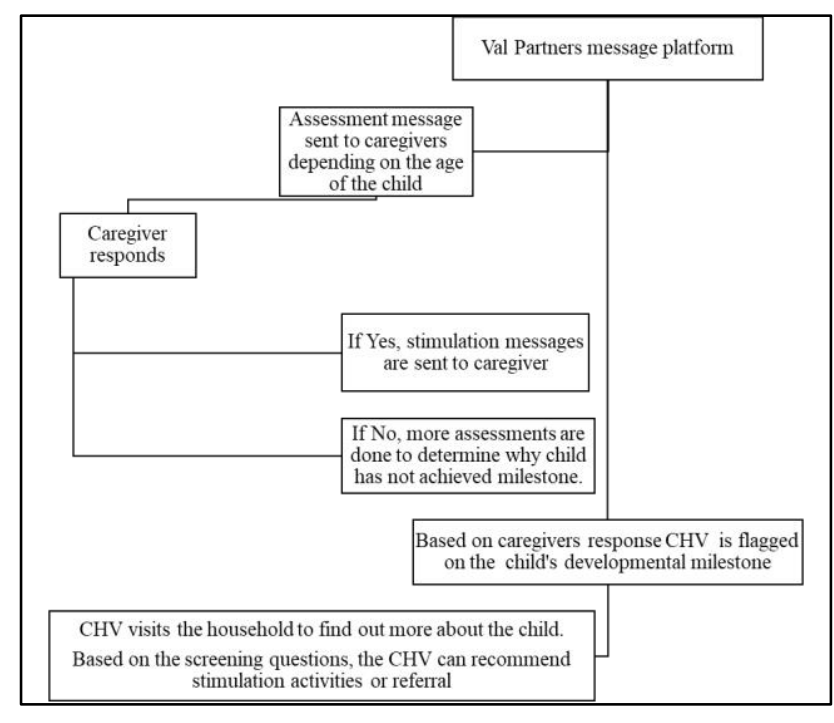

Figure 1: Implementation process.

\section{Data collection procedures}

\section{Recruitment and training of data collection field workers}

We will recruit and train two research teams. The quantitative team will collect quantitative data including socio-demographic data and infants' developmental scores at baseline, mid-line, and end-line. The qualitative interviewers will conduct in-depth interviews and focus group discussions with caregivers, $\mathrm{CHVs}$, sub-county and county personnel. The teams will be trained on the general study procedures including ethical issues, consenting, and specific procedures in which each will be involved. Data quality, accuracy and completeness will be emphasized.

\section{Qualitative data}

Qualitative interviews will be conducted with caregivers, $\mathrm{CHVs}$, policy makers/implementers, healthcare managers and providers at the national, county and sub-county level as appropriate, to determine their perceptions on the intervention including its importance, feasibility and efficiency, among other aspects. This will help in assessing whether the stakeholders will value the program, that is, whether they think the program will meet their needs.

Focus group discussions with $\mathrm{CHV}$ s and caregivers of children under two years will help in understanding their needs and potential solutions to such needs. Qualitative interviews will be held with both female and male caregivers to establish the feasibility of using the mobile application in tracking children's developmental outcomes, their role in the project, perceived benefits of the intervention, and facilitators and hindrances to their 
participation in caregiving activities. In addition, key informant interviews with members of the sub-county health management team and providers of early childhood development (ECD) services will help in understanding the gaps in early identification of developmental delays and how the program will address the identified gaps.

CHVs will be interviewed on their experience with the mobile application, their perceptions on the feasibility of its use to track children's developmental progression, and their perceptions on the support they provide to caregivers using the mobile application to track children's developmental progression.

Information on caregiving knowledge and practices will be obtained at baseline (before the intervention starts), midline (halfway through the intervention) and at endline (after a period of twelve months of the intervention) to measure change. Data on operational feasibility of the mobile phone technology plus home-based ECD intervention will be collected at baseline, midline and end-line.

\section{Quantitative methods}

Quantitative questionnaires will be used to establish the use of the mobile application. Data will be collected through interviews with both mothers and fathers about their experiences with the mobile phone technology, and with caregiving. Caregiver knowledge, attitudes and practice (KAP) will be measured using a set of questions that cover topics such as appropriate care, feeding practices, play and communication activities and learning activities likely to promote holistic development in children. Caregiving knowledge will be assessed through maternal report of the different aspects of child development. We will use ten questions on caregiver ECD knowledge which will be scored on a Likert scale ranging from ' 1 ' =agree completely to ' 4 ' $=$ disagree completely.

Caregiving practices will be assessed by maternal selfreport of their responsiveness and the opportunities for age-appropriate play and learning available to the child at home. The mother/caregiver will also be asked about their involvement with the child, acceptance and provision of learning materials. Trained field workers will administer the protocol for child monitoring - infanttoddler version (PCM-IT) to assess children's developmental outcomes. The items on the PCM-IT are derived from tools including the Kilifi developmental inventory, the developmental milestones checklist and the profile for socioemotional development. Information on children's functioning is obtained through a combination of caregiver self-reported questions and direct observations, similar to the procedures used in a study conducted in Kilifi, Kenya. ${ }^{15}$ These tools were validated among young children in rural communities and they exhibited community acceptability.
At baseline, we will also administer the ages and stages questionnaire -third edition (ASQ-3) to establish children's 'developmental' age before the start of the intervention. ${ }^{16}$ This information will be used to determine the starting level for the first and subsequent messages that will be sent to the caregivers. For instance, with regards to motor development, if the child is able to 'sit on his/her own,' the messages that will be sent to the caregiver at the first contact will be those where the mother will be encouraged to stimulate the development of the next milestone, that is, crawling. The ASQ-3 is used to measure children's developmental outcomes in terms of their gross and fine motor skills, language, socio-emotional and physical development through a combination of primary caregiver self-reported questions and direct observations. ${ }^{17}$ The ASQ-3 is a globally used primary caregiver-reported, easy-to-use, reliable, and validated screening instrument for identifying potential developmental delays among children aged between two and 60 months.

\section{Data analysis}

Qualitative data will be transcribed into Microsoft Word files and imported into NVivo software to identify primary and meta codes and major themes. Analysis across transcripts will be done thematically.

Differences in caregiver KAP and wellbeing at baseline and end-line for the intervention and control groups will be determined by summing the scores on the items and running a t-test for comparison.

For child developmental outcomes, raw scores will be constructed by adding all the items passed for each domain on the PCM-IT. Composite scores will be computed by summing the scores across all the domains. As we expect scores on each test to increase with age, the scores will be standardized for age to allow for direct comparisons across tests. After the data are cleaned, quantitative data analysis will be performed using Stata. A first set of analyses will consist of descriptive statistics. This will allow us to detect similarities and/or differences between subjects' characteristics across the different groups. We will compare some baseline measurements between the control group and intervention group using the t-test adjusted for continuous variables, and clusteradjusted chi-square for binary variables. The second set of analysis will consist of assessing the causal effect of the ECD intervention in Korogocho via the DID estimator, and mixed linear models. Individual items on the PCM-IT will be reviewed for missing data, as well as for floor and ceiling effects. In the case where more than $10 \%$ of the responses on the items are missing, the data will be excluded from further analysis. The time taken to complete the assessment will be calculated. We will also calculate the totals by summing the scores of the items included in the tool to establish children's performance levels at baseline and at end-line. This will enable us to 
identify child development domains that have acceptable variability.

\section{Ethical considerations}

The study will be conducted after review by the APHRC's internal scientific review committee has been completed and ethics approval was obtained from the Amref Health's Ethics and Scientific Review Committee (ESRC) and the National Commission for Science Technology and Innovation (NACOSTI). The investigators have undergone training in protection of human subjects in research, and the National Institutes of Health (NIH) web-based training course on the protection of human participants. They are therefore cognizant of the fundamental principles in research on human participants: respect for persons, beneficence, nonmalevolence and justice. Field-workers will also receive training on research ethics. We shall seek informed consent from all study participants. For those who cannot read, the information sheet will be read to them in English or Kiswahili and they will be asked to provide a thumb print to signify their consent in presence of a witness.

\section{DISCUSSION}

The project examines the feasibility and impact of utilizing mobile phone technology to enable caregivers to track/monitor their children's development in real time. The innovation requires that users (caregivers) have access to a phone to receive and respond to the monthly text messages about children's milestones. Given that about $80 \%$ of the general population in Kenya has access to a mobile phone, we expect that even in a very poor community like Korogocho, a large proportion of the population will be eligible to participate in this project.

During the implementation, we will capture information on perceptions of caregivers and CHVs on the use of mobile phone technology to track children's development, and their experiences with the intervention. We will engage CHVs to follow up caregivers who may be slow in responding to the messages. If this intervention proves feasible and is seen to have impact, we expect that CHVs should be able to support it at the community level.

We are working with a low income population for whom affordability of airtime may be a challenge. Although the cost of sending the text messages will be borne by the project throughout its duration, one of the considerations that we will need to make in the scale-up is the participants' willingness to pay. This project is expected to generate useful information that will inform the scaling up of the mobile phone technology to other counties in Kenya, and other resource-limited settings in SSA. Involvement of the county health and education representatives and $\mathrm{CHVs}$ throughout the project is expected to promote buy-in and support for the sustainability of the innovation.

\section{ACKNOWLEDGEMENTS}

We are grateful to the Saving Brains, Grand Challenges Canada for funding this project. We thank the Val partners for their support in the designing of the innovation. We appreciate the APHRC investigator team for all their input, and we thank the Amref, NACOSTI and the wider County governance and the local community for their input.

Funding: Saving Brains, Grand Challenges Canada, grant number SB-POC-1810-20425

Conflict of interest: None declared

Ethical approval: The study was approved by the Amref Health Africa Ethics and Scientific Review Committee in Kenya (ESRC P578/2018), and by NACOSTI

\section{REFERENCES}

1. Awiti PO, Grotta A, Van Der Kop M, Dusabe J, Thorson A, Mwangi J, et al. The effect of an interactive weekly mobile phone messaging on retention in prevention of mother to child transmission (PMTCT) of HIV program: Study protocol for a randomized controlled trial (WELTEL PMTCT). BMC Med Inform Decis Mak. 2016;16:1-8.

2. House D, Cheptinga P, Rusyniak D. Availability of mobile phones for discharge follow-up of pediatric Emergency Department patients in western Kenya. Peer J. 2015;3:e790.

3. Zurovac D, Sudoi RK, Akhwale WS, Ndiritu M, Hamer DH, Rowe AK, et al. The effect of mobile phone text-message reminders on Kenyan health workers' adherence to malaria treatment guidelines: A cluster randomised trial. Lancet. 2011;378(9793):795-803.

4. Harwood R, Leyendecker B, Carlson V, Asencio M, Miller A. Handbook of parenting volume 4: Social conditions and applied parenting. J Marriage Family. 2002.

5. Black MM, Walker SP, Fernald LCH, Andersen CT, DiGirolamo AM, Lu C, et al. Early childhood development coming of age: science through the life course. Lancet. 2017;389:77-90.

6. Meuwissen AS, Carlson SM. Fathers matter: The role of father parenting in preschoolers' executive function development. J Exp Child Psychol. 2015;140:1-15.

7. Kimani-Murage EW, Fotso JC, Egondi T, Abuya B, Elungata P, Ziraba AK, et al. Trends in childhood mortality in Kenya: The urban advantage has seemingly been wiped out. Heal Place. 2014;29:95103.

8. Kimani-Murage EW, Wekesah F, Wanjohi M, Kyobutungi C, Ezeh AC, Musoke RN, et al. Factors affecting actualisation of the WHO breastfeeding 
recommendations in urban poor settings in Kenya. Matern Child Nutr. 2015.

9. Zahn-Waxler C, Shirtcliff EA, Marceau K. Disorders of Childhood and Adolescence: Gender and Psychopathology. Annu Rev Clin Psychol. 2008;4:275-303.

10. Abubakar A, Holding P, van Baar A, Newton CRJC, van de Vijver FJR. Monitoring psychomotor development in a resource-limited setting: An evaluation of the Kilifi Developmental Inventory. Ann Trop Paediatr. 2008;28(3):217-26.

11. Richter LM, Daelmans B, Lombardi J, Heymann J, Boo FL, Behrman JR, et al. Investing in the foundation of sustainable development: pathways to scale up for early childhood development. Lancet. 2017;389:103-118.

12. National Bureau of Statistics Nairobi K. Republic of Kenya Kenya Demographic and Health Survey, 2014.

13. Beguy D, Ndugwa R, Kabiru CW. Entry into motherhood among adolescent girls in two informal settlements in Nairobi, Kenya. J Biosoc Sci. 2013;45(6):721-42.

14. Faul F, Erdfelder E, Lang AG, Buchner A. G*Power 3: A flexible statistical power analysis program for the social, behavioral, and biomedical sciences. In: Behavior Research Methods. 2007;39:175-91.

15. Abubakar A, Holding P, Van De Vijver F, Bomu G, Van Baar A. Developmental monitoring using caregiver reports in a resource-limited setting: The case of Kilifi, Kenya. Acta Paediatr Int J Paediatr. 2010;99(2):291-7.

16. Squires J, Bricker D. Ages \& Stages Questionnaires. Third Edition. (ASQ-3): A Parent-Completed ChildMonitoring System. Paul Brookes Publ Co; 2009.

17. Hsiao C, Richter L, Makusha T, Matafwali B, van Heerden A, Mabaso M. Use of the ages and stages questionnaire adapted for South Africa and Zambia. Child Care Health Dev. 2017;43(1):59-66.

Cite this article as: Wekulo PK, Nampijja M, Okullo DO, Okelo KO, Njeri M, Onyango S, et al. A quasiexperimental study on exploring the use of mobile phone technology for optimizing, tracking and responding to children's developmental progress in Korogocho, Nairobi, Kenya: study protocol. Int J Clin Trials 2020;7(1):43-9. 